\title{
Enjoying Breathing Program: A National Prospective Study Protocol to Improve Chronic Obstructive Pulmonary Disease Management in Chinese Primary Health Care
}

This article was published in the following Dove Press journa

International Journal of Chronic Obstructive Pulmonary Disease

\author{
Cunbo Jia ${ }^{\mathrm{l}, *}$ \\ Chunyu Zhang ${ }^{1} *$ \\ Fang Fang ${ }^{1, *}$ \\ Ke Huang ${ }^{2}$ \\ Fen Dong $\mathbb{1 D}^{3}$ \\ Xiaoying $\mathrm{Gu}^{3}$ \\ Hongtao Niu (iD ${ }^{2}$ \\ Shan $\mathrm{Li}^{4}$ \\ Chen Wang ${ }^{2,5}$ \\ Ting Yang ${ }^{2}$
}

\begin{abstract}
'Department of Health Reform and Development, China-Japan Friendship Hospital, Beijing, People's Republic of China; ${ }^{2}$ Department of Pulmonary and Critical Care Medicine, China-Japan Friendship Hospital; National Clinical Research Center for Respiratory Diseases; Institute of Respiratory Medicine, Chinese Academy of Medical Science, Beijing, People's Republic of China; ${ }^{3}$ Data Platform, China-Japan Friendship Hospital, Beijing, People's Republic of China; ${ }^{4}$ School of Management, Beijing University of Chinese Medicine, Beijing, People's Republic of China; ${ }^{5}$ Chinese Academy of Medical Sciences and Peking Union Medical College, Beijing, People's Republic of China
\end{abstract}

*These authors contributed equally to this paper

Correspondence: Ting Yang

Department of Pulmonary and Critical

Care Medicine, China-Japan Friendship

Hospital; National Clinical Research

Center for Respiratory Diseases; Institute of Respiratory Medicine, Chinese

Academy of Medical Science, Chaoyang

District, Beijing 100029, People's Republic of China

Tel +86/0-84205907

Email dryangting@qq.com
Background: Chronic obstructive pulmonary disease (COPD) is prevalent and poses a heavy burden worldwide. However, patients know little about COPD, and primary health care providers have poor therapy capability in China. Enjoying Breathing Program aims to establish a new comprehensive COPD patient management system, including early detection, standardized therapy, and follow-up in China. The goal of the study is to 1) describe the intervention for physicians and patients and 2) to assess the effectiveness of this program.

Methods: It is the first nationwide trial involving all levels of health care institutions from primary health care institutions to tertiary hospitals. It includes a series of structured but individualized intervention for both health care providers and COPD patients. Primary health care providers from pilot hospitals will take both online and face-to-face courses, including the procedure of COPD patients' management and prevention, diagnosis and treatment. Once the patients are diagnosed with COPD, they will undertake standard therapy and self-management education program, perform rehabilitation exercises, and be followed up by primary health care providers every 3 months. The primary outcome will be exacerbation-related hospital/emergency admission and the change of patients' awareness and primary health care providers' knowledge of COPD within 36 months. Secondary outcome will include the change of pulmonary function test, structured COPD patients' management, two-way referral, and standardized therapy.

Conclusion: A comprehensive COPD patient management model to promote the standardized therapy will be established; this will improve COPD patients' awareness and health quality.

Trial Registration Number: This study has been registered at www.ClinicalTrials.gov (registration identifier: NCT04318912).

Keywords: protocol, national prospective trial, COPD management

\section{Background}

\section{COPD Prevalence Internationally and in China}

Chronic obstructive pulmonary disease (COPD) is characterized by persistent airflow limitation and is a highly prevalent chronic disease that affects nearly 174.5 million adults worldwide in $2015 .^{1-3}$ The most recent Chinese national survey of COPD was carried out between June 2012 and May 2015. It involved 50,991 adults aged 20 years or older, and reported an overall prevalence of spirometry-defined COPD at $8.6 \%$. Prevalence was higher in people aged $\geq 40$ 
years than in those aged 20-39 years. This prevalence is tantamount to 100 million people in China affected by COPD. ${ }^{4}$

COPD occurs progressively, and the airflow limitation is not fully reversible. COPD patients experience frequent exacerbations, poorer health status, accelerated decline in forced expiratory volume in one second (FEV1), worsened quality of life and, increased hospital admissions and mortality. ${ }^{3,5}$ It is estimated by World Health Organization that COPD will become the third leading cause of death and seventh leading cause of disability-adjusted life years (DALYs) by $2030 .^{6-9}$

\section{Low Awareness of COPD Among Patients}

The level of disease knowledge and self-management behaviors of COPD patients is relatively low in China. ${ }^{10}$ A national survey reported that among spirometry-defined COPD patients in China, only $2.6 \%$ were aware of their condition, $9.7 \%$ of the adult population had done pulmonary function examination previously, and only $12.0 \%$ of the people with COPD and $55.8 \%$ of those with a self-reported history of COPD had pulmonary function examination previously.

Evidence from systematic reviews of research involving patient education have identified an association between structured but individualized patient education (including self-management intervention) and improvements in health-related quality of life, lower probability of respirator-related hospital admissions, shorter hospital stays and more appropriate use of medication for exacerbations, compared with participants who received usual care. ${ }^{11-13}$ The intervention strategies are targeted to help patients manage the disease more effectively, ${ }^{13-15}$ through pulmonary rehabilitation exercise classes, smoking cessation, maintenance therapy, and understanding of COPD symptoms. However, there are few national researches about Chinese COPD patients' education and management intervention carried out in China.

\section{Poor Capability for COPD Patient Management Among Primary Health Care Providers}

COPD is a chronic disease, and the primary health care providers are in charge of the patient's management. However, compared with hypertension and diabetes, primary health care providers know less about COPD. As a result, their ability to prevent, diagnose, and treat it is relatively poorer. ${ }^{16,17}$ The main reason for poor management ability is lack of training. It is reported that only one third of primary health care providers received COPD knowledge training in the past three years. ${ }^{18}$

\section{The Enjoying Breathing Program}

The Enjoying Breathing Program is designed by Medical Cluster for Respiratory Diseases and National Center for Respiratory Diseases Clinic Research. It is aimed to establish a new comprehensive COPD patients' management model involving all levels of health care institutions. This program teaches physicians about prevention, early detection, and treatment of COPD as well as follow up of COPD patients every three months. Meanwhile, it also educates COPD patients about self-management (preventing exacerbation) and taking decision in different disease situations. Finally, primary health care providers acquire the ability to handle COPD, and patients change their health behaviors to manage COPD.

\section{Objectives of the Study Protocol}

This paper described the protocol of the Enjoying Breathing Program and it determines the effectiveness in establishing the new comprehensive COPD patient management system.

We hypothesize that the program, which aims to improve the ability of primary health care physicians and the patients' adherence to therapy using multiple strategies (educational information, self-management and reminders), will result in less readmission, early detection, improved COPD patients' treatment and management.

\section{Methods and Analyses}

The study is a prospective and open study carried out in multi centers. The medical file of each patient addressed to the Enjoying Breathing Program center will be screened. Patients will be included in the study: 1) if they meet inclusion criteria and 2) if they provide their signed informed consent.

\section{Study Population}

Primary Health Care Providers

Inclusion criteria:

- Those who work in the pilot regions

Exclusion criteria: 
- Absent from clinic work more than 6 months in the past year

\section{Patients}

Inclusion criteria:

- Aged $\geq 40$ years

- Post-bronchodilator FEV1/FVC $\leq 70 \%$ or confirmed COPD patients

- Previously diagnosed by physicians but not under standard management

- Local residents who will be accessible and available during trial

- Written informed consent

Exclusion criteria:

- Patients with physician diagnosis of asthma or asthma-COPD overlap syndrome

- Patients hospitalized for COPD within previous one month

- Having severe cognitive dysfunction and is unable to communicate

- Severely ill with less than 12-month life expectancy

- Having participated in similar trials or are undergoing other clinical trials

- Patient refuses

\section{Sample Size}

One of the important measures is to compare the change of the knowledge of primary health care providers after continued medical education. We will apply Dunnett test to compare the changes from baseline. Due to our pilot study the standard deviation is 2.78 , the expected improvement is 2 ; it is regarded significant. If the $\alpha$ is 0.05 and the $\beta$ is 0.1 , the minimum health care worker sample size is 17 at very time point. ${ }^{19}$ However, all the health institutions will participate in this program in each pilot region. A minimum of 200 of them from each pilot region will take the COPD Knowledge Test, given that there are average 50 health institutions in a pilot region.

Our primary goal is less exacerbation-related hospital/ emergency admission. Repeated measure will be used to compare the change before intervention, and at 12 months, 24 months, and 36 months after intervention. We set $\alpha$ as 0.05 and $\beta$ as 0.1 . The standard deviation is 1.05 and the intraclass correlation coefficient (ICC) is 0.78 , according to the result of pilot study. We hope the annual number of exacerbations could be reduced at least once. The minimum patient sample size is $10 .^{20}$ However, the original aim is that more COPD patients will benefit from this program. Given the COPD prevalence rate of $13.7 \%$ among patients aged $\geq 40$ years and with at least 110 thousand people aged $\geq 40$ years in each pilot region, it estimated that at least 10,000 participants will be screened and at least 600 eligible patients will be followed up in each pilot region. To address a request from local authorities to invite all residents in the pilot region to be involved in this community-based study, the maximum number of participants is invited to participate.

\section{Design}

\section{Step I - Pilot Regions Selection}

29 regions were selected randomly after being stratified by their economic rank. The support from local authority is thought to be key to the Enjoying Breathing Program. As a result, all the health care institutions are required to join the program after the 29 local authorities issue the documents showing their permits and support to the program.

\section{Step 2 - Continued Medical Education}

Early diagnosis and intervention for COPD patients are thought to be key to preventing exacerbation. Once the local authority issues document permitting and supporting the Enjoying Breathing Program, central team members will begin the field instruction on COPD patient screening and management. Later, physicians or nurses from pilot hospitals will regularly receive continued medical education both online and on field. National online continued medical education, including diagnosis, treatment and preventing exacerbation of COPD (especially how to test pulmonary function and follow up patients), will be given by famous Chinese COPD specialists. The electronic study video can be reviewed online at any time. Meanwhile, the local leading pilot hospitals will oversee monthly field continued medical education.

To examine the effectiveness of our continued medical education, it will be combined with the COPD Knowledge Test which is a 30-item questionnaire (please see Appendix 1) and completed on-line by primary health care providers at the baseline, 12 months, 24 months and 36 months after intervention respectively. During the pilot test, the Physician's Questionnaire on COPD showed good internal consistency (Cronbach's $\alpha=0.80$ ) and reliability (retest reliability $=0.90$ ). A minimum of 200 primary health care providers from each pilot region will take the test. 


\section{Step 3 - High-Risk COPD Patients Screening by Questionnaire}

The COPD Screening Questionnaire (COPD-SQ) was designed by Ran Pixin. The 7 items are measures of high-risk population (please see Appendix 2). The sensitivity, specificity and correct classification rates of this questionnaire were $60.6 \%, 85.2 \%$ and $82.7 \%$, at a cut-off score of 16 respectively. ${ }^{21}$ If the score is more than 16 , the person will be regarded as high-risk population and take pulmonary function test for further diagnosis.

Besides screening by questionnaire, participants need to fill the patient COPD Knowledge Questionnaire (please see Appendix 3) at baseline, 12 months, 24 months and 36 months after intervention respectively. The Patient COPD Knowledge Questionnaire was designed by us. The Cronbach's $\alpha$ and the retest reliability estimated through pilot test are 0.80 and 0.88 respectively.

\section{Step 4 - Diagnosed by Pulmonary Function Test}

The portable pulmonary function machines have been allocated by central team of Enjoying Breathing Program. If the FEV1/FVC is $<70 \%$, patients will inhale bronchodilators and have the pulmonary function test again. If the value of FEV1/FVC is still $<70 \%$, and other pulmonary diseases, such as the asthma, pneumonectasis etc. could be excluded, the patients could be diagnosed as COPD.

In this step, patients' medical records are established including the disease history, family history, recent examination results, and the first time diagnosed as COPD. COPD assessment test (CAT) questionnaire (please see Appendix 4), modified Medical Research Council (mMRC), ${ }^{22}$ and EQ-5D-5L, ${ }^{23}$ which are widely used to evaluate the COPD patients' health related life, are also applied in the program. CAT, which has good internal consistency (Cronbach's $\alpha=0.88$ ) and test-retest reliability (intra-class correlation coefficient 0.8 ), is an 8 -item questionnaire for assessing and monitoring COPD. ${ }^{24} \mathrm{mMRC}$ is considered adequate for assessment COPD symptoms and is widely used for COPD patients. ${ }^{25}$ EQ-5D-5L, developed recently, measures health using five levels of severity in five dimensions: mobility, self-care, usual activities, pain/discomfort, and anxiety/depression. The EQ-5D-5L has been widely used in both general population and patient samples with 1.87 absolute discriminatory power and 0.81 relative discriminatory power compared with EQ-5D-3L. ${ }^{26}$

\section{Step 5 - Regular and Continuous Intervention}

Patients will receive a multifaceted and integrated disease management. There will be monthly COPD education course, including but not limited to appropriate medication use (eg antibiotics and oral steroids for exacerbations), inhaler use, and visiting physicians immediately after symptoms appear. The patients will be treated or transferred depending on their individual situation. In case patients are not using inhaler correctly, more education on appropriate inhaler use will be conducted. Additionally, smoking cessation counseling will be provided to patients who are active smokers, and aged patients will be persuaded to receive influenza/pneumococcal vaccine immunization. Patients will also be encouraged to exercise regularly, including 8-week pulmonary rehabilitation program. Trained physicians will contact patients monthly to monitor their symptoms, review, and adjust medications if needed. Guidance on maintenance therapy (LAMA, LABA, ICS) will be delivered by physicians every three months.

\section{Step 6 - Patients Following Up}

Patients need to visit physicians at least once a month for maintenance therapy, because the max dose for chronic disease is one month. Every three months primary health care providers will interview the COPD patient via CAT, mMRC and EQ-5D-5L questionnaire to learn the health-related life and record the history of exacerbation, the result of pulmonary function test, medication and referring history. Every year, the COPD patients will be invited to refill the COPD Knowledge Questionnaire. If the patient does not come in after half a month, the physician will try to keep in touch with the patients to remind them. If the patient could not be contacted, the reason why they could not be contacted is recorded in our online COPD patient's management system.

Please see Figure 1 for the whole study process.

\section{Intervention}

\section{Continued Medical Educations}

The primary health care providers from 29 pilot regions have access to e-materials at any time, including the following five areas: (1) the risk factors and prevalence of COPD, (2) how to diagnose and treat COPD, (3) how to prevent COPD exacerbation, (4) how to test pulmonary function, (5) the procedure of the Enjoying Breathing Program, including how to use the online COPD patient management system.

Physicians who pass both the face-to-face exam taken by local leading hospital and the online test are qualified to participate in Enjoying Breathing Program. The continued medical educations will last the whole period of our 


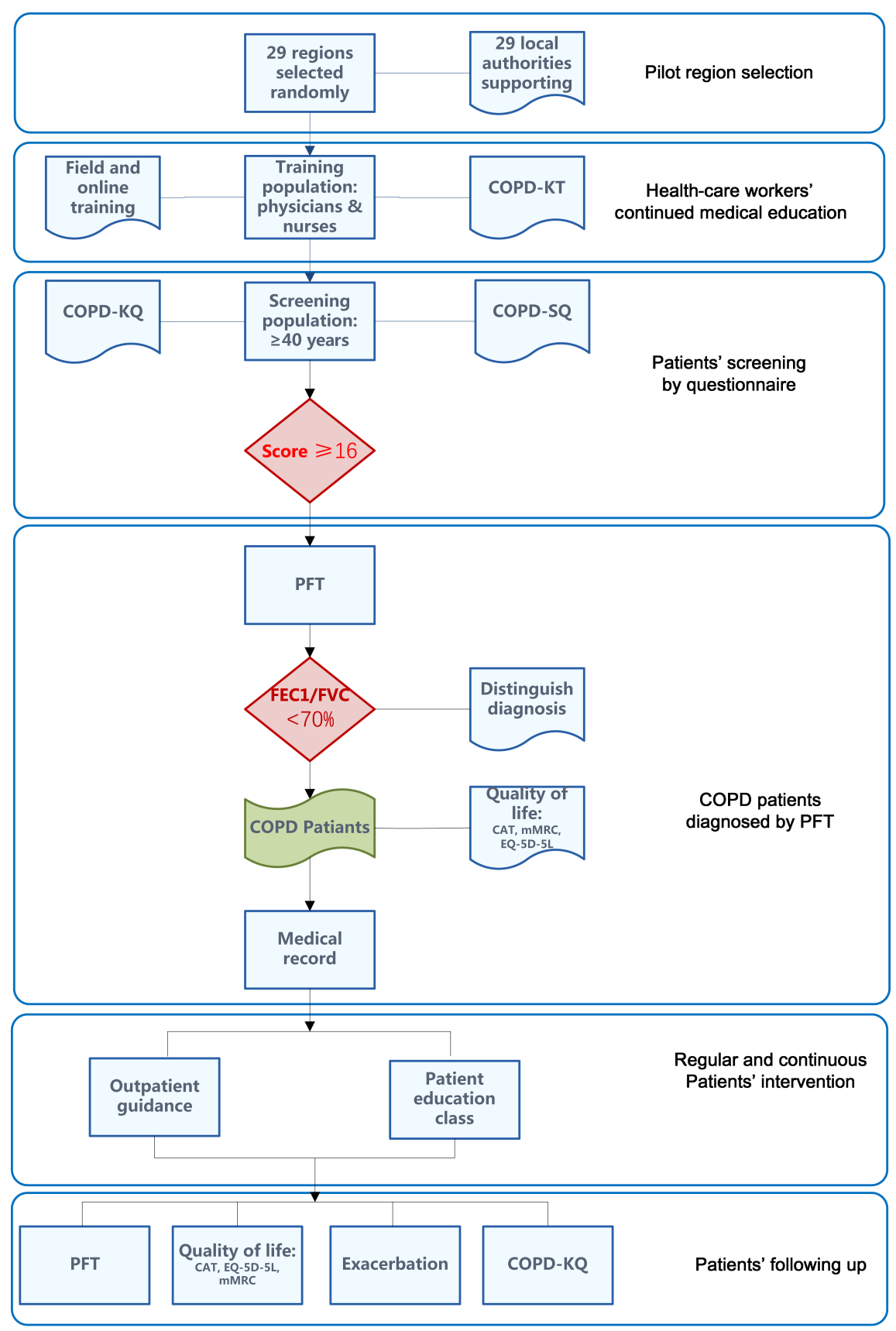

Figure I Study flow Chart.

Abbreviations: COPD-KT, COPD Knowledge Test; COPD-KQ, COPD Knowledge Questionnaire; COPD-SQ, COPD Screening Questionnaire; PFT, pulmonary function test; CAT, COPD assessment test; mMRC, modified Medical Research Council.

program; meanwhile the study material will be updated as necessary.

\section{Self-Management Interventions}

Self-management interventions are structured but personalized and multi-component. Appropriate actions include contacting physicians for support, initiating self-treatment and maintenance therapy, prevention plan (for example, smoking cessation and influenza vaccination), rehabilitation program and sending reminders to participants (for example, to get maintenance therapy or get the flu vaccine). The interventions will also last from the beginning to the end point.

\section{Acceptability and Feasibility Study}

Physician intervention delivery using digital technology has resulted in users having the ability to access intervention 
support when they require it or when they are available. Faceto-face course taken by local leading hospital provides interaction between experts and primary health care providers.

The structured active patient COPD health education provided by huge number of primary health care providers will improve engagement and anticipate a range of physician reactions. Online management system has also been developed and connected with the system of pulmonary function machine. This system not only captures pulmonary function data in time, it reminds physicians to follow up patients on time.

All the data collection by primary health care providers will be investigated by central research team to ensure the quality and progress of the study.

\section{Outcome Measures}

The efficacy of Enjoying Breathing Program to improve management and self-management of COPD patients will be assessed using a series of standardized direct and indirect outcome measures. The primary and most important outcome measure will be the exacerbation-related hospital/emergency admission, the change of patients' awareness and primary health care providers' knowledge of COPD within 36 months. All other outcome measures will be secondary.

\section{Exacerbation-Related Hospital/Emergency Admission Within 36 Months}

We will compare exacerbation-related hospital/emergency admissions before intervention, and at 12 months, 24 months, and 36 months after intervention.

Meanwhile, the time to first exacerbation, the severity of first exacerbation and the number of exacerbations during follow up will be analyzed. 30-day and 90-day readmission rate due to exacerbation all cause readmission rates, and non-COPD related readmission rates will be compared before intervention and at 12, 24, and 36 months after intervention. The differences in length of stay for exacerbation among the 4 periods will also be tested.

\section{Change of Awareness and Self-Management of COPD Patients}

The patient Knowledge Form is an 11-item questionnaire (please see Appendix 3) completed by COPD patients four times before intervention, and at 12, 24, and 36 months after intervention respectively. These forms assess patients' awareness of COPD. We will compare the changes before and after intervention, as well as the visiting rates for maintenance therapy at 12,24 , and 36 months from baseline.

\section{The Change of the Knowledge of Primary Health} Care Providers

The primary health care provider Knowledge Test is a 29item questionnaire (please see Appendix 1) completed by primary health care providers at the baseline, 12, 24, and 36 months respectively. These forms assess the knowledge including risk factors, prevention, detection, and therapy of COPD. We will compare the trend of the change of COPD knowledge among primary health care providers at 12,24 , and 36 months after intervention from baseline.

\section{Change of Pulmonary Function Test Within 36 Months}

Given the importance of early detection of COPD, pulmonary function test has been recommended into routine health examination especially for population aged 40 and older. ${ }^{27}$ We will compare the change of the percentage of those taking pulmonary test among the population aged 40 and older visiting primary health care clinics at 12,24 , and 36 months from baseline.

\section{Change of COPD Patients' Structured Management}

As mentioned before, COPD patients' structured management includes routine maintenance therapy, monthly health education, and follow up every 3 months etc. We will analyze the ratio of the number of those undertaking the structured management by primary health care providers, to COPD patients diagnosed by pulmonary function test. Simultaneously, we will also compare the change of the ratio of COPD patients' structured management at 12 , 24, and 36 months from baseline.

\section{The Change of Two-Way Referral Rate}

Different health care institutions have different functions. Primary health care institutions are responsible for the rehabilitation and daily management of COPD patients, while secondary and tertiary hospitals are responsible for the diagnosis of COPD and the treatment of exacerbation. One of the goals of this program is to integrate primary health care institutions and secondary and tertiary hospitals to manage COPD patients together. Thus, we will also analyze two-way referral rates in terms of the proportion of patients who are referred to higher or lower level health care institutions in the total number of outpatient and inpatients with COPD in the year. The two-way referral rate before intervention will be compared with that at 12 , 24, and 36 months, respectively. 


\section{The Change of COPD Patients' Standard Treatment Rate}

2017 Global Initiative for Chronic Obstructive Lung Disease (GOLD) guidelines are used to determine whether a treatment is standardized. The percentage of the number of COPD patients undertaken standard treatment in the total number of COPD patients who visit the hospital in the year will be compared at 12, 24, and 36 months from baseline.

\section{Recruitment and Consent}

Primary health care providers are contacted by local health authorities and have mandatory continued medical education. Those who pass the examination will get a personal account on the COPD patient management system.

Patients will be contacted via their family doctors or community workers and invited to participate. Those who agree to participate, will be sent consent forms, will fill COPD screening questionnaires, and further have pulmonary function test and followed up by primary health care providers. Recruitment will continue until a minimum of 600 patients have been achieved at each pilot regions. It is expected that this sample size will enable data saturation to be achieved to understand the feasibility and acceptability of the developed intervention.

\section{Trial Status}

The trial is currently at the stage of primary health care provider continued medical education. Patient data will be collected between May 2020 and April 2023.

\section{Process Evaluation}

Questionnaires designed by the research team.

\section{Statistical Analysis}

All analyses will be conducted using SAS 9.4 (SAS Institute Inc., Cary, North Carolina, USA). A two-sided $\mathrm{p}$ value less than 0.05 will be considered to indicate statistical significance. All continuous variables will be measured using standard measures of central tendency, with mean and standard deviation or median and IQR, according to statistical distribution.

Student's $t$-test or Mann-Wilk test will be applied to compare the primary outcome- times of exacerbation before and after intervention. A multivariable analysis will then be performed using Cox analysis to incorporate adjustment on possible confounders covariates selected according to clinical relevance and to univariate results.
Multivariable analysis results will be presented using regression coefficients and 95\% CI. For categorical outcomes (such as readmission rate, all causes readmission rate, and non-COPD related readmission rate etc.), $\chi^{2}$ or Fisher's exact test will be used. At last, mixed effect model will be performed to compare the trend changes of patients' self-management behavior and primary health care providers' ability.

\section{Ethics and Dissemination}

Ethics approval has been granted by China-Japan friendship hospital (approval number 2019-41-k29). This study will be conducted in accordance with ethical principles of the Declaration of Helsinki. All participants will provide written informed consent. The findings will be submitted for publication in peer-reviewed scientific journals and presented at international scientific conferences.

\section{Discussion}

Enjoying Breathing program is a national multicenter trial that is aimed to establish a new COPD patient management model through the structured primary care workers education and patient self-management intervention. The present protocol describes the procedure of Enjoying Breathing program and will examine the effect of our intervention.

\section{Strengths}

COPD is a progressive disease and impose high costs on healthcare systems. ${ }^{28}$ It is known as a major driver of decline in health status, considering a set of system physical function limitations including poor musculoskeletal strength and function, poor exercise performance and selfreported functional limitations due to COPD. ${ }^{29}$ Patient education has proven beneficial effect on health-related quality of life, and lower risk for respiratory-related hospital admission compared with those who received usual care. However, there is little research to prove that this is also effective in China. To our knowledge this is the first national multicenter study to use a community-based structured intervention to detect, treat and manage COPD patients, with a large targeted population (135 million), and health institutions (more than 3000).

Furthermore, Enjoying Breathing Program is under the support and allowance of 29 local authorities, with all the health care institutions in the regions actively involved.

Finally, it is a longitudinal study requiring 3 years to acquire better COPD awareness among patients and 
knowledge among primary health care providers, eventually promoting standardized management and improved outcome for patients.

\section{Limitations}

Patients' adherence to these interventions is one of the biggest challenges we will face. If their adherences are low, it may lead to smaller improvement from baseline. To improve patient adherence, we will screen those who have good adherence in run-in period. Eligible patients will be well-informed of the goal and design of this study. Patients will be timely contacted by primary health care providers to receive education session, use medications, record daily symptoms, and so on.

\section{Conclusion}

In summary, this study, Enjoying Breathing Program, a large national trial, will address if a strategy of structured patient education and primary health care providers continue medical education can improve COPD patients' awareness and health quality, as well as promote the standardized therapy.

\section{Data Sharing Statement}

Data sharing is not applicable to this article as no datasets will be generated or analyzed during the current study.

\section{Acknowledgments}

The authors would like to thank authorities from 29 pilot regions for including Enjoying Breathing Program in their government action plan. We also would like to thank Chunhe Da, Yudong An, Jianyong Zhang, Quanxian Liu, Feng Xu, Aibin Deng, Yanping Lv, Chunxiang Pan, Aihua Hou, Shuchun Sun, Guoru Yang, Lingling Tan, Xiaoling Wang, Su Tang, Lijun Chen, Yanhong Liu, Mingyan Jiang, Huan Liu, Ying Li, Lixiao Li, Jie Cao, Jinna Li, Wenshu Chai, Yan Zhou, Wen Cheng, Zhengguo Zhang, Huiming Yin, Mudan Zhou, Zhihua Xiao, Yafang Yang, Li Li, Zhiqiang Dong, Ruifeng Shi etc. for their contribution to the implementation of this program.

\section{Author Contributions}

All authors made substantial contributions to conception and design; took part in drafting the article and revising it critically for important intellectual content; gave final approval of the version to be published; and agree to be accountable for all aspects of the work.

\section{Funding}

This work was supported by National Key R\&D Program of China (2016YFC1303900), CAMS Innovation Fund for Medical Sciences (CIFMS) (2018-I2M-1-001) and Enjoying Breathing Program is funded by GSK exclusively.

\section{Disclosure}

The authors report no conflicts of interest in this work.

\section{References}

1. Vos T, Allen C, Arora M; Collaborators GDaIIaP. Global, regional, and national incidence, prevalence, and year lived with disability for 310 diseases and injuries, 1990-2015: a systematic analysis for the Global Burden of Disease Study 2015. Lancet. 2016;388:1545-1602. doi:10.1016/S0140-6736(16)31678-6

2. Soriano JB, Abajobir AA, Abate KH; Collaborators GCRD. Global, regional, and national deaths, prevalence, disability-adjusted life years, and year lived with disability for chronic obstructive pulmonary disease and asthma, 1990-2015: a systematic analysis for the Global Burden of Disease Study 2015. Lancet Respir Med. 2017;5:691-706. doi:10.1016/S2213-2600(17)30293-X

3. Halbert RJ, Isonaka S, George D, Iqbal A. Interpreting COPD prevalence estimates: what is the true burden of disease? Chest. 2003;123:1684-1692. doi:10.1378/chest.123.5.1684

4. Wang C, Xu J, Yang L, et al. Prevalence and risk factors of chronic obstructive pulmonary disease in China (the China Pulmonary Health [CPH] study): a national cross-sectional study. Lancet. 2018;391:1706-1717. doi:10.1016/S0140-6736(18)30841-9

5. Mannino DM. A Sonia B. Global burden of COPD: risk factors, prevalence, and future trends. Lancet. 2007;370:765-773. doi:10. 1016/S0140-6736(07)61380-4

6. Chan KY, Li X, Chen W, et al. Prevalence of chronic obstructive pulmonary disease (COPD) in China in 1990 and 2010. J Glob Health. 2017;7:020704. doi:10.7189/jogh.07.020704

7. Miravitlles M, Ribera A. Understanding the impact of symptoms on the burden of COPD. Respir Res. 2017;18:67. doi:10.1186/s12931017-0548-3

8. Yin P, Wang H, Vos T, et al. A subnational analysis for mortality and prevalence of chronic obstructive pulmonary disease in China 1990-2013: findings from Global Burden of Disease Study (GBD) 2013. Chest. 2016;150:1269-1280. doi:10.1016/j.chest.2016.08.1474

9. Murray CJ, Lopez AD. Alternative projections of mortality and disability by cause 1990-2020: global burden of disease study. Lancet. 1997;349:1498-1504. doi:10.1016/S0140-6736(96)07492-2

10. Yang H, Wang H, Du L, Wang Y, Wang X, Zhang R. Disease knowledge and self-management behavior of COPD patients in China. Medicine. 2019;98:e14460. doi:10.1097/MD.0000000000014460

11. Howcroft M, Walters EH, Wood-Baker R, Walters JA. Action plans with brief patient education for exacerbations in chronic obstructive pulmonary disease. Cochrane Database Syst Rev. 2016;12: CD005074. doi:10.1002/14651858.CD003091.pub4

12. Russell S, Ogunbayo OJ, Newham JJ, et al. Qualitative systematic review of barriers and facilitators to self-management of chronic obstructive pulmonary disease: views of patients and healthcare professionals. Npj Prim Care Respir Med. 2018;28:2. doi:10.1038/ s41533-017-0069-z

13. Korpershoek YJ, Slot JCB, Effing TW, Schuurmans MJ, Trappenburg JC. Self-management behaviors to reduce exacerbation impact in COPD patients: a Delphi study. Int J Chron Obstruct Pulmon Dis. 2017;12:2735-2746. doi:10.2147/COPD.S138867 
14. Boer GMD, Mennema TH, Noort EV, Chavannes NH, Birnie E, Veen J. Intrinsic factors influence self-management participation in COPD: effects on self-efficacy. Erj Open Res. 2018;4:00011-2018. doi:10.1183/23120541.00011-2018

15. Guan WJ, Ran PX, Zhong NS. Prevention and management of COPD in China: successes and major challenges. Lancet Respir Med. 2016;4:428-430. doi:10.1016/S2213-2600(16)30092-3

16. Zhang RB. Awareness of knowledge of COPD by Doctors in District and Community Hospitals. Chin J Prev Control Chron Non-Commun Dis. 2009;17(1):61-63.

17. Bo ZP P, Sinan W, Yong Z, et al. Chronic respiratory disease management in grassroots medical institutions of China. Chin Gen Pract. 2018;21:1513-1520

18. XU Yang ZP, Ting Y, Chen W. Current status of prevention and control of chronic obstructive pulmonary disease in primary care in China. Chin Gen Pract. 2016;19:4153-4158.

19. Machin D, Campbell MJ, Tan SB, Tan SH. Sample Size Tables for Clinical Studies. 3rd ed. Wiley-Blackwell; 2011.

20. Liu H, Wu T. Sample size calculation and power analysis of time-averaged difference. J Mod Appl Stat Meth. 2005;4:434-445. doi:10.22237/jmasm/1130803680

21. Zhou YM, Chen SY, Tian J, et al. Development and validation of a chronic obstructive pulmonary disease screening questionnaire in China. Int J Tuberc Lung Dis. 2013;17:1645-1651. doi:10.5588/ ijtld.12.0995

22. Jones PW, Harding G, Berry P, Wiklund I, Chen WH, Leidy NK. Development and first validation of the COPD assessment test. Eur Respir J. 2009;34:648-654. doi:10.1183/09031936.00102509
23. Mahler DA, Wells CK. Evaluation of clinical methods for rating dyspnea. Chest. 1988;93:(3):580-586.

24. Herdman M, Gudex C, Lloyd A. et al. Development and preliminary testing of the new five-level version of EQ-5D (EQ-5D-5L). Quality of Life Research. 2011;20:(10):1727-1736.

25. Thomsen M, Nordestgaard BG, Vestbo J, Lange P. Characteristics and outcomes of chronic obstructive pulmonary disease in never smokers in Denmark: a prospective population study. Lancet Respir Med. 2013;1:543-550. doi:10.1016/S2213-2600(13)70137-1

26. Janssen MF, Pickard AS, Golicki D, et al. Measurement properties of the EQ-5D-5L compared to the EQ-5D-3L across eight patient groups: a multi-country study. Qual Life Res. 2013;22:1717-1727. doi:10.1007/s11136-012-0322-4

27. National Health and Family Planning Commision of the People's Republic of China. The 13th Five Year Plan for Healthcare; 2016. Available from: http:/www.gov.cn/zhengce/content/2017.01/10/con tent 5158488.htm. Accessed January 1, 2020.

28. Han MLK. Clinical correlations of computed tomography imaging in chronic obstructive pulmonary disease. Ann Am Thorac Soc. 2013;10: S131-S137. doi:10.1513/AnnalsATS.201303-046AW

29. Eisner MD, Blanc PD, Yelin EH, et al. COPD as a systemic disease: impact on physical functional limitations. Am J Med. 2008;121:789-796. doi:10.1016/j.amjmed.2008.04.030

\section{Publish your work in this journal}

The International Journal of COPD is an international, peer-reviewed journal of therapeutics and pharmacology focusing on concise rapid reporting of clinical studies and reviews in COPD. Special focus is given to the pathophysiological processes underlying the disease, intervention programs, patient focused education, and self management protocols. This journal is indexed on PubMed Central, MedLine and CAS. The manuscript management system is completely online and includes a very quick and fair peer-review system, which is all easy to use. Visit http://www.dovepress.com/testimonials.php to read real quotes from published authors 